Federal Reserve Bank of Minneapolis

Research Department Staff Report 400

November 2007

\title{
Lifetime Aggregate Labor Supply with Endogenous Workweek Length*
}

\author{
Edward C. Prescott \\ Federal Reserve Bank of Minneapolis \\ and Arizona State University \\ Richard Rogerson \\ Arizona State University \\ Johanna Wallenius \\ Arizona State University
}

\begin{abstract}
This paper studies lifetime aggregate labor supply with endogenous workweek length. Such a theory is needed to evaluate various government policies. A key feature of our model is a nonlinear mapping from hours worked to labor services. This gives rise to an endogenous workweek that can differ across occupations. The theory determines what fraction of the lifetime an individual works, not when. We find that constraints on workweek length have different consequences for total hours than total labor services. Also, we find that policies designed to increase the length of the working life may not increase aggregate lifetime labor supply.
\end{abstract}

*Prescott thanks the National Science Foundation (Grant 0422539), Rogerson thanks the National Science Foundation (Grant 0417822, and Wallenius thanks the Yrjo Jahnsson Foundation for supporting the research. We also thank our colleagues with whom we discussed this research. We want to thank Simona Cociuba for providing exceptional research assistance. The views expressed herein are those of the authors and not necessarily those of the Federal Reserve Bank of Minneapolis or the Federal Reserve System. 
As an empirical matter, the amount of labor supplied by an individual over his or her lifetime is effectively characterized by two numbers: the fraction of lifetime spent in employment, and the hours worked when employed. In this paper we develop a tractable dynamic general equilibrium model that delivers this characterization as an equilibrium outcome and present some of its implications for policy analysis and data interpretation.

The key feature of our model is a nonlinear mapping from hours of work in a given period to labor services provided in that same period. Specifically, at low hours of work this mapping is convex, due to such factors as the costs associated with getting set up in a job, communicating with coworkers, meeting with supervisors, and so on. At high hours of work this mapping is assumed to be concave, due to fatigue. ${ }^{1}$ Similar to Hornstein and Prescott (1993), this nonlinearity implies that workweeks of different lengths are not perfect substitutes in generating labor services, but does so in a much more tractable manner.

We embed this feature of technology into a dynamic model populated by a continuum of identical individuals and study efficient allocations and competitive equilibrium outcomes for this economy. Two key findings emerge. First, efficient allocations are completely characterized by two numbers: the fraction of lifetime spent in employment by each individual, and hours worked while employed. Second, although this economy possesses an important nonconvexity, we show that efficient allocations can be achieved as competitive equilibria with only a standard set of Arrow-Debreu markets: markets for consumption and labor services at each date. In particular, there is no need to allow for trade in lotteries or sunspots in order to implement efficient

\footnotetext{
${ }^{1}$ Rosen (1978) also noted that this formulation was a tractable way to think about intensive and extensive margins of labor supply.
} 
allocations, as in Prescott and Townsend (1984a, 1984b), Rogerson (1988), or Shell and Wright (1993).

To illustrate the usefulness of the model, we use it to consider the effects of several policies: a tax and transfer policy, a restriction on workweek, and a restriction on fraction of lifetime devoted to work. Several interesting findings emerge. First, the model implies a large aggregate labor supply elasticity in response to tax and transfer programs, and at the same time it predicts a very small (in fact, zero) elasticity for hours of work of continuously employed individuals. In this regard, the model mimics the implications of a model that simply assumes labor is indivisible. A key message is that in our model, the aggregate labor supply elasticity with respect to changes in taxes is a function not only of preference parameters, but also of technology parameters. Specifically, features of the mapping from hours of work to units of labor services are critical in determining the aggregate labor supply elasticity.

A second finding is that any distortion to one component of lifetime labor supply will be at least partially offset by movements in the other component. Increasing working life by changing the nature of social security benefits, for example, will lead to a decrease in the workweek. This has important implications for how policy makers forecast budget implications associated with social security reform. Third, distortions to lifetime labor supply can also lead to changes in hours of work and labor services in opposite directions. For example, we show that a decrease in the workweek can lead to greater aggregate hours of work but lower output.

An additional implication of an exogenous restriction on workweek length in our model is that the equilibrium wage per hour of work necessarily decreases. In practice, many governments that adopt workweek restrictions in an attempt to increase 
employment (and thereby total hours of work) also simultaneously adopt policies that lead to higher wages per hour of work. When the hourly wage rate is not allowed to fall, it no longer follows that a restriction on hours will necessarily increase aggregate hours of work. This is consistent with the findings of the empirical literature.

Any theory of workweek length should be able to account for the simple observation that workweek length differs significantly across occupations. By emphasizing the role of technology as a determinant of optimal workweek length, our theory provides a natural explanation for such differences. We illustrate this by developing a two-occupation version of our model with endogenous occupational choice. This extension provides an interesting implication about the relationship between labor supply elasticities estimated from a cross-section of workers and the aggregate elasticity associated with a change in taxes. Specifically, in our model the cross-section elasticity of hours with respect to wages produces an estimate of the preference parameter that determines the curvature in the disutility of work function. However, because of the nonconvexity in technology, this preference parameter is irrelevant for the response of aggregate hours to the tax and transfer program we study.

An outline of the paper follows. Section 1 presents some motivating observations from the data. Section 2 introduces the nonconvex technology in a static setting, and Section 3 analyzes equilibrium in the dynamic setting. Section 4 presents the policy analysis, and Section 5 considers the extension to a two-occupation setting. Section 6 concludes. 


\section{Motivating Facts}

In this section we present five facts concerning labor supply over the lifetime that serve as motivation for the model that we develop in the next section. The first two facts concern changes in the nature of work over the life cycle for individuals with at least a moderate attachment to the labor force. We consider the cutoff for this to be working more than 800 hours during the year, which is just over 15 hours per week.

Fact 1: In the United States, the fraction of people working at least 800 hours declines significantly with age.

The supporting evidence is shown in Figure 1, which shows the fraction of individuals working more than 800 hours per year as a function of age, based on the 2004 CPS.

Figure 1

People Working at Least 800 Hours by Age, U.S. 2004

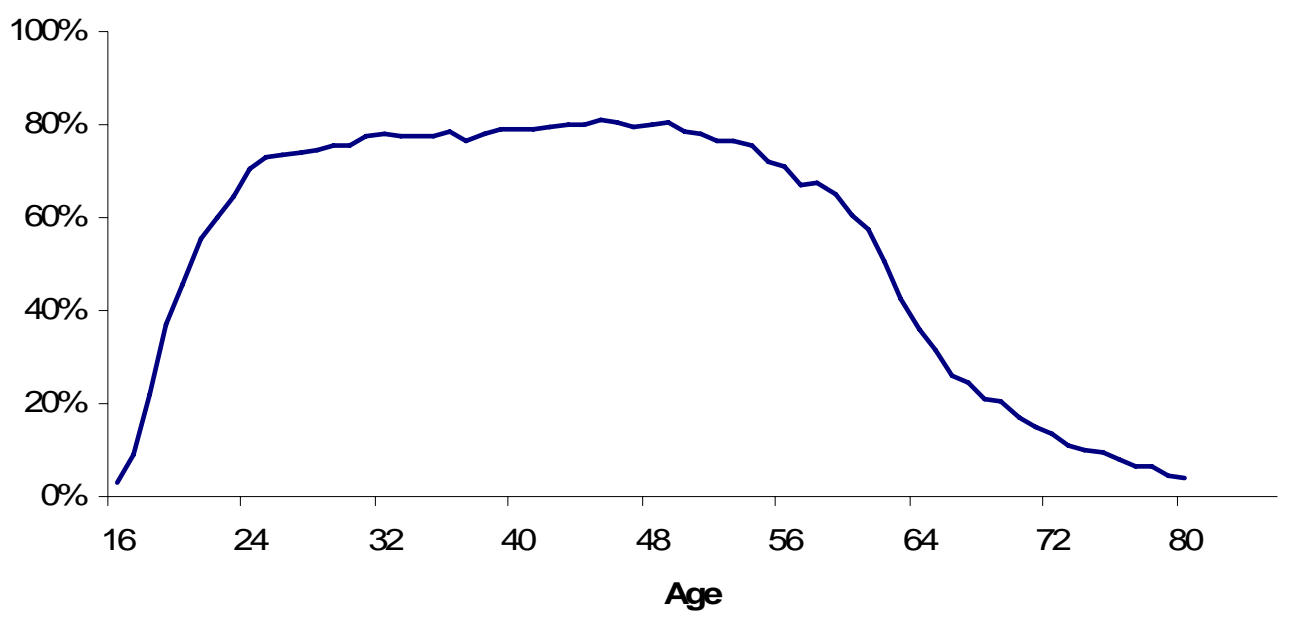

Source Data: CPS 2004 
Note that the fraction of individuals working above this threshold level begins to decrease in the late 40 s and falls relatively smoothly through to the early 70 s, at which point it flattens out somewhat. This value drops by roughly a quarter between the ages of 48 and 60.

The next fact considers what happens to the amount of work performed by those individuals working more than 800 hours per year.

Fact 2: In the United States, hours worked per person for those individuals who work at least 800 hours per year declines little with age.

The supporting evidence is shown in Figure 2, which plots annual hours of work conditional on working at least 800 hours per year, again using data from the CPS for 2004.

Figure 2

Hours Worked by Those Who Work More Than 800 Hours, U.S. 2004

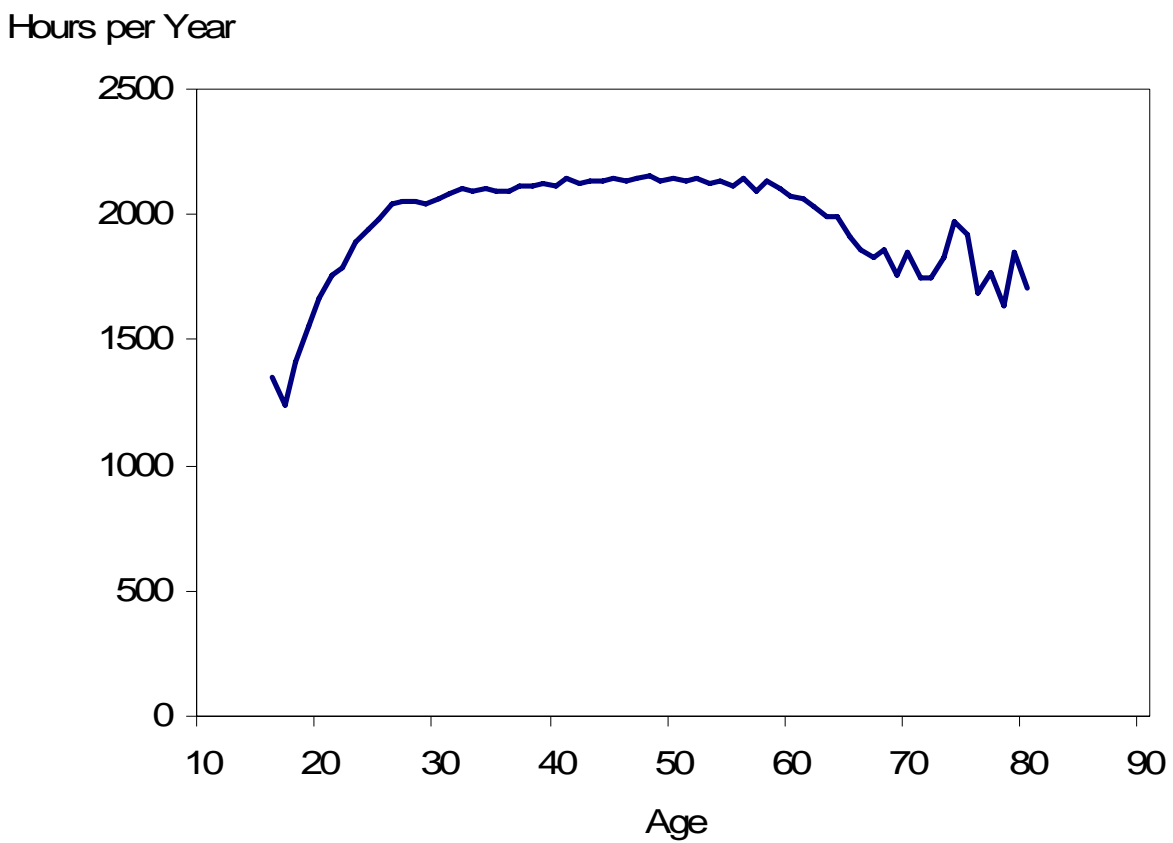

Source Data: CPS 2004 
Note that the decrease in hours is much smaller than the decrease in the fraction of individuals working more than 800 hours per year. Moreover, the hours series is relatively flat beyond age 60 . Taken together, Facts 1 and 2 imply that changes along the extensive margin dominate both the decrease in hours for those above 50 and the increase in hours worked for younger workers. Put somewhat differently, the fraction of life spent in employment seems a key margin.

The next fact notes the importance of this margin in understanding differences in hours of work across countries.

Fact 3: About half the difference in hours worked between the United States and continental Europe is accounted for by differences in the fraction of lifetime worked.

This is shown in Figure 3.

\section{Figure 3}

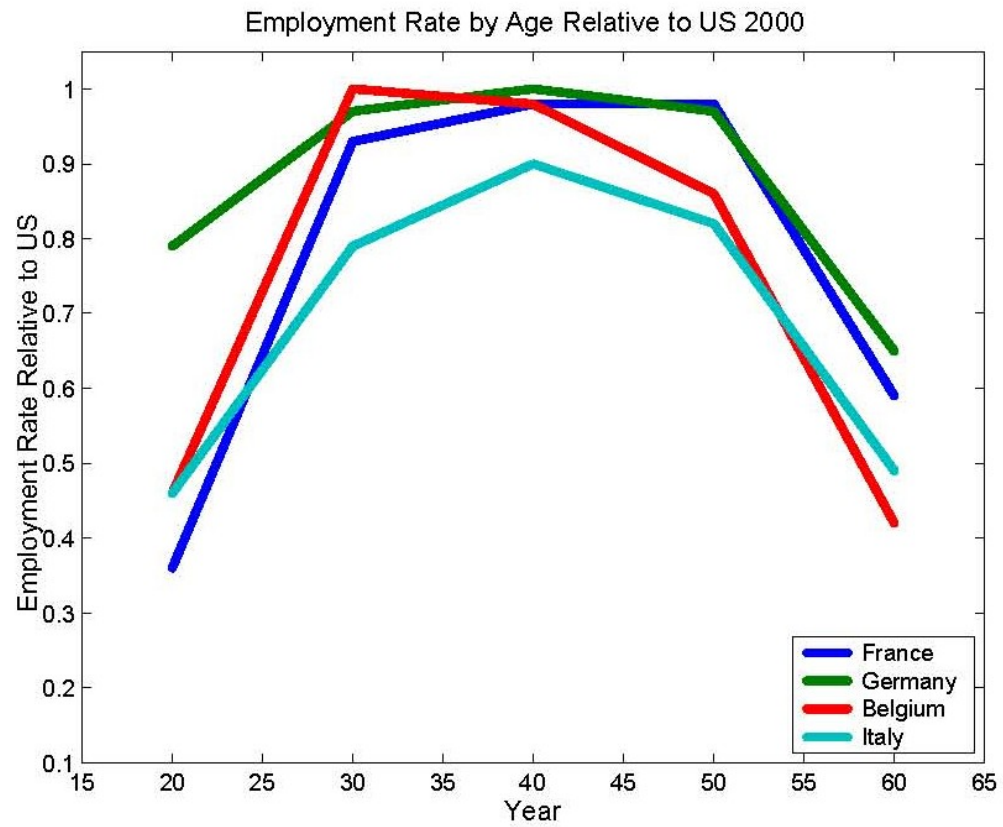

Source: OECD Database 
This figure shows relative employment rates for five age groups (15-24, 25-34, etc....) for four countries in continental Europe relative to the United States. Note that for primeaged individuals the relative employment rate is approximately one, and that it decreases quite significantly at younger and older ages. The important message here is that individuals in these European countries spend a much smaller fraction of their life in employment.

Although our analysis will not focus on business cycle movements, the next fact notes that the analogous finding carries over to this context as well.

Fact 4: Cyclically in the United States the principal margin of adjustment is the fraction of the working age population employed. Hours worked per worker in a given week vary little.

Cho and Cooley (1994) establish this fact. They find that three-quarters of the variation in total hours of employment takes the form of movements in and out of the labor force rather than adjustments in average hours of work.

The last fact that we document is that there are significant differences in hours of work across groups of workers.

Fact 5: There are sizable differences in workweek lengths across categories of workers based upon occupation and education.

Table 1 highlights the differences across occupations. 
Table 1

Weekly Hours Worked for Selected Occupations*

\begin{tabular}{|l|l|l|}
\hline Less than 40 Hours & Between 40 and 45 Hours & More than 45 Hours \\
\hline $\begin{array}{l}\text { Food Preparation and } \\
\text { Serving (36.11) }\end{array}$ & Legal (44.76) & Management (45.98) \\
$\begin{array}{l}\text { Building and Grounds } \\
\text { Maintenance (38.96) }\end{array}$ & $\begin{array}{l}\text { Education, Training and } \\
\text { Library (40.76) }\end{array}$ & $\begin{array}{l}\text { Farming, Fishing and } \\
\text { Forestry (45.09) } \\
\text { Office and Administrative } \\
\text { Support (38.87) }\end{array}$ \\
$\begin{array}{l}\text { Healthcare Practitioners } \\
\text { and Technical (40.21) }\end{array}$ & Extraction Workers (51.30) \\
& Sales (41.29) & \\
& Construction (41.95) & \\
& Production (41.40) & \\
\hline
\end{tabular}

* Includes people working more than 800 hours per year.

Source Data: CPS 2004

\section{Static Model of Intensive and Extensive Margins}

Two key messages emerge from the previous section. First, the fraction of life devoted to employment is a key margin of labor supply for an individual. Second, the choice of hours conditional on employment seems to respond to features of the economic environment such as those associated with differences in occupation. In this section we develop a static model that delivers intensive and extensive margins of work as part of its equilibrium. In the next section we embed this model in a dynamic setting to produce a model in which the fraction of life devoted to work is a key margin, and in which features of technology can influence the choice of hours along the intensive margin.

We assume a continuum of identical individuals who derive utility from consumption of a single good $(c)$ and leisure $(1-h)$. Time devoted to market work is 
denoted by $h$, and each individual has a unit time endowment. For simplicity we consider a utility function that is separable between consumption and leisure, so preferences are described by the von Neumann-Morgenstern utility function:

$$
u(c)-\tilde{v}(h)
$$

where $u(c)$ is strictly increasing, strictly concave, and twice continuously differentiable, and $\tilde{v}(h)$ is strictly increasing, strictly convex, and twice continuously differentiable. We also normalize $\tilde{v}$ so that $\tilde{v}(0)=0$.

There is a constant returns to scale technology that uses labor services $(L)$ to produce the single consumption good. We normalize the marginal product to one and hence write the production technology as

$$
C=L,
$$

where uppercase letters denote aggregates. It is standard to assume that an individual's input of labor services is either equivalent to their time devoted to market work, or more generally proportional to their time devoted to market work. Central to our model is the notion that time devoted to work is not the same as input of labor services. In particular, we assume that the mapping from individual time devoted to market work to input of labor services is nonlinear and write

$$
l=g(h)
$$

where $l$ is the quantity of labor services yielded by an individual who supplies $h$ units of time. The function $g$ is assumed to be increasing and twice continuously differentiable, with $g(0)=0$, but is first convex and then concave. In particular, we assume that there is some $\bar{h} \in(0,1)$, such that $g^{\prime}$ is weakly increasing on $[0, \bar{h})$ and weakly decreasing on $[\bar{h}, 1]$. Figure 4 shows a particular function with these properties. 
Figure 4

\section{Mapping from Hours of Work to Labor Services}

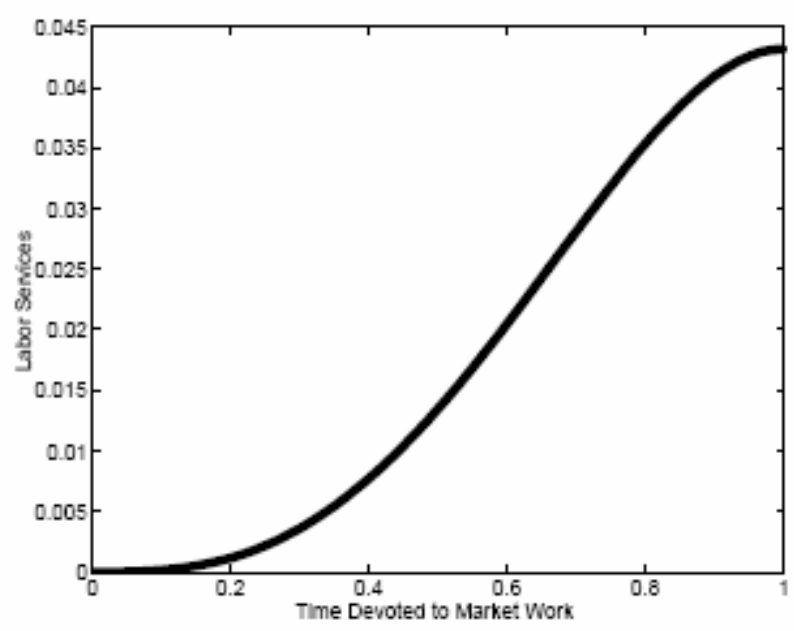

The $g$ function captures two key economic features. The first is the possibility that long hours of work lead to fatigue, so that past some point additional units of time input lead to lower increments in labor services. This is captured by the fact that $g$ may be strictly concave over the interval $[\bar{h}, 1]$. The second economic feature that is captured by the $g$ function is that for a range of hours worked, replacing one worker who supplies $h$ units of time with two workers who each supply .5h units of time implies a lower input of labor services. In reality, a variety of factors contribute to this effect, including such things as set-up costs, supervisory time, and coordination issues. While for some issues it may be important to explicitly model these underlying factors, we believe that embedding them directly into the $g$ function is a useful and powerful abstraction. Note that this last property of $g$ is consistent with the observation that for many activities, firms do not consider part-time workers. $^{2}$

\footnotetext{
2 There are, of course, some activities for which firms prefer part-time workers, due to factors such as the timing of demand. Our analysis can accommodate differences across activities by allowing the $g$ function to differ across occupations or sectors. We present a two-occupation example in Section 5.
} 
The properties that we have embedded in $g$ have important implications for the form that efficient resource allocations take in this economy. In particular, if we had assumed that $g$ was simply the identity function, then (equal weight) efficient allocations in our economy would have the property that all individuals devote the same time to market work and enjoy the same consumption. This result would continue to hold if we assumed that $g$ were everywhere concave. However, this is not necessarily the case when the $g$ function contains a convex region as we have allowed.

To pursue this issue, it is convenient to consider a change in variables. In particular, rather than considering the nonconvexity in the technology, we consider a transformation that places the nonconvexity in preferences. To do this, rather than considering preferences defined over consumption and time devoted to market work, we instead define preferences over consumption and the supply of labor services. This is easily accomplished, since there is a one-to-one mapping between time devoted to market work and the supply of labor services. Letting $g^{-1}$ denote the inverse function of $g$, we now define preferences as

$$
u(c)-v(l),
$$

where

$$
v(l)=\tilde{v}\left(g^{-1}(l)\right)
$$

In order to have a nicely behaved $v$ function, we impose some additional structure on the $\tilde{v}$ and $g$ functions. Specifically, we assume that $\frac{\tilde{v}^{\prime \prime}}{\tilde{v}^{\prime}}$ is weakly increasing and that $\frac{g^{\prime \prime}}{g^{\prime}}$ is 
weakly decreasing. ${ }^{3}$ Given our assumptions on $g$ and $\tilde{v}$, it follows that $v$ is strictly increasing and twice continuously differentiable, that $v(0)=0$, and that $v$ is initially concave and then later convex. Figure 5 shows a particular function with these properties.

Figure 5

\section{Disutility from Labor Services}

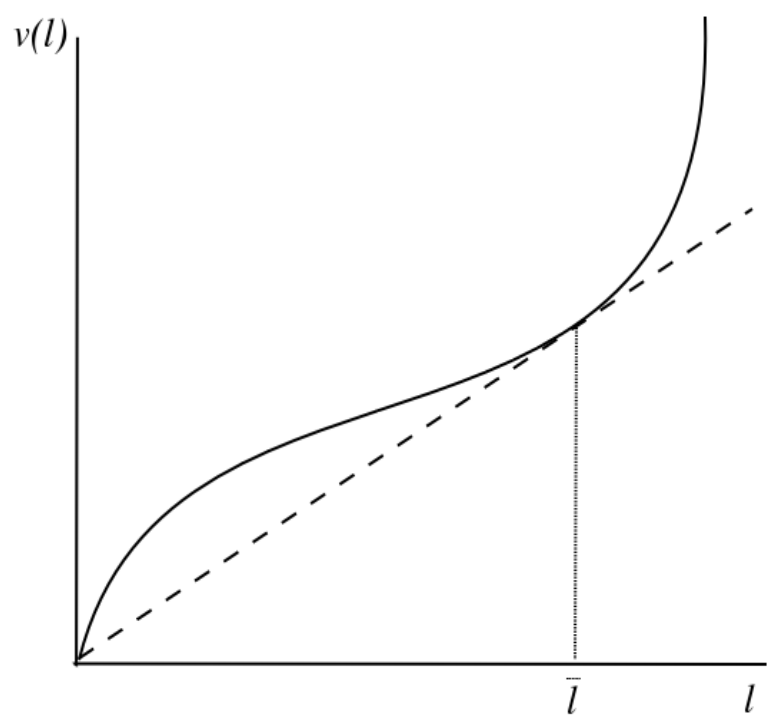

The two formulations of the problem are analytically equivalent, but the labor services formulation allows for a simple graphical exposition. Figure 5 geometrically illustrates why the nonconvexity of the $g$ function may affect the form of efficient allocations. The dashed line in this figure is the steepest line passing through the origin, which is no greater than $v(l)$ at all points. This line has a point of tangency with the $v(l)$ curve at $\bar{l}$. It is straightforward to show that it would never be optimal to have workers supply labor services less than $\bar{l}$, which necessarily translates into the statement that it would never be optimal for all workers to supply less than $h$ hours, where $\bar{l}=g(h)$. The

\footnotetext{
${ }^{3}$ This assumption can actually be weakened. For our purposes, it is enough to assume that $\frac{\tilde{v}^{\prime \prime}}{\widetilde{v}^{\prime}}$ and $\frac{g^{\prime \prime}}{g^{\prime}}$ cross 1 at most.
} 
reason for this is simple: suppose that all workers were to supply labor services less than $\bar{l}$. Then by placing appropriate fractions of individuals at 0 and $\bar{l}$, a social planner can have the same aggregate input of labor services, but the average disutility associated with obtaining this given labor input will be less, since the dashed line lies everywhere below the $v(l)$ curve for $l \in(0, \bar{l})$.

More generally, one can show that the optimal allocation will take one of two forms: either all individuals supply the same labor services, and this value is at least $\bar{l}$, or there is one group of individuals that supplies 0 units of labor services and another group that supplies $\bar{l}$ units of labor services. Strict concavity of $u(c)$ implies that a Social Planner who is maximizing an equal weighted integral of individual utilities would necessarily allocate consumption evenly across all individuals, independently of the labor services provided by a given individual. In the case where not all individuals supply positive units of labor services, the solution to this Social Planner's problem does not determine which individuals supply positive units of labor services, but only the fraction of individuals who supply positive units.

It is straightforward to show that this Social Planner's problem has a unique solution for the fraction of individuals who work (e), the labor services provided by those who work ( $($ ), and consumption of all individuals $(c)$. Recalling that $v(0)=0$, these values are solutions to the following problem:

$$
\begin{gathered}
\max _{c, e, l} u(c)-e v(l) \\
\text { s.t. } c=e l, 0 \leq e \leq 1,0 \leq l \leq g(1)
\end{gathered}
$$


The interesting case is the one where the solutions for $e$ and $l$ are both interior. In this case, after substituting the resource constraint into the objective function, one obtains the following two first-order conditions for $e$ and $l$, respectively:

$$
\begin{aligned}
& u^{\prime}(e l) l=v(l) \\
& u^{\prime}(e l)=v^{\prime}(l)
\end{aligned}
$$

Dividing these two equations by each other gives

$$
\frac{v(l)}{l}=v^{\prime}(l) .
$$

This condition says that the optimal level of labor services per worker should equate the marginal disutility per unit of labor services to the average disutility per unit of labor services. Geometrically, this is the property that $\bar{l}$ possessed in Figure $5 .{ }^{4}$ Note that this condition does not depend on the level of $e$, as long as the solution for $e$ is interior. Given a solution for $l$, it is then straightforward to use either Eq. (6) or Eq. (7) to solve for the optimal value of $e$. Given that $l$ is determined, the value of time devoted to market work is easily determined by inverting the function $g$.

The allocation just described implies that not all individuals will devote the same number of hours to market work, but that all individuals will receive the same consumption. As a result, this allocation cannot be achieved as a competitive equilibrium unless lotteries or sunspot contracts are considered. The fact that decentralizing optimal allocations requires randomization has been seen by some as an argument against focusing on these allocations. In the next section we show that once one moves to a

\footnotetext{
${ }^{4}$ From Figure 5 one can see that there are actually two values of $l$ for which this condition holds, but the second-order conditions for an optimum are violated at the lower value, since it occurs in the region where $v$ is concave rather than convex.
} 
lifetime setting as opposed to a static setting, optimal allocations can be decentralized without resorting to any randomization.

\section{Lifetime Labor Supply}

In this section we extend the static analysis of the previous section to a dynamic context in which individuals live for many periods. For reasons that will become clear soon, we model time as continuous and assume that the horizon of the economy is normalized to 1 , so that time runs continuously from 0 to 1 . As noted previously, we can use either the hours of work or the units of labor services formulation for our analysis. Here we choose to focus on observables, and thus present the results for the hours of work formulation.

All of the primitives are identical to those from the previous section, and lifetime utility is given by

$$
\int_{0}^{1}[u(c(t))-\tilde{v}(h(t))] d t
$$

when we consider the consumption space to be consumption and time devoted to market work. We have assumed no discounting to simplify the presentation, but this is not essential to the arguments.

We continue to assume that there is no capital in the economy, so that labor services are the only input into production. This implies that

$$
Y(t)=L(t)
$$

at each point in time, where capital letters denote aggregates.

While it remains true that one can generate efficient allocations (i.e., allocations that maximize an equal weighted integral of utilities) by simply applying the random 
allocation of the previous section at each instance of time, it turns out that one can also achieve the same outcome in utility space without resorting to any randomization. In particular, rather than having the Social Planner randomly choose a fraction $e$ of individuals to work at each instance, the Social Planner can instead have each individual work for a fraction $e$ of their lifetime, and then simply coordinate these choices across individuals so that at each point in time the fraction of individuals working is also equal to $e$. It is straightforward to see that these two allocations are both feasible and that they both generate the same expected utility to individuals.

The importance of this observation is that it also tells us that we can decentralize efficient allocations without resorting to any randomization. In particular, consider the setting in which the commodity space consists of consumption and labor services supplied. We consider an Arrow-Debreu equilibrium, in which markets for consumption and labor services at all dates exist at time zero, and denote the price of consumption and labor services at time $t$ by $p(t)$ and $w(t)$, respectively. ${ }^{5}$ Given the linear technology, competitive equilibrium requires that $w(t)=p(t)$ at each instant. It is easy to show that in equilibrium the price $p(t)$ must be constant over time, in which case we can normalize all prices to one.

With this normalization, the problem of each consumer in equilibrium is given by

$$
\begin{gathered}
\max _{c(t), h(t)} \int_{0}^{1}[u(c(t))-\tilde{v}(h(t))] d t \\
\text { s.t. } \int_{0}^{1} c(t) d t=\int_{0}^{1} g(h(t)) d t, 0 \leq h(t) \leq 1, c(t) \geq 0
\end{gathered}
$$

\footnotetext{
${ }^{5}$ We note for future reference that in this economy, the wage per unit of labor services is not the same as the wage per unit of time.
} 
But this problem is exactly identical to the problem that the Social Planner solves in the static model, so that the individual choice problem can be recast as choosing a fraction $e$ of his or her lifetime to work and the hours of work $h$ to be supplied at those instances when he or she works. That is, each individual solves

$$
\begin{gathered}
\max _{c, e, l} u(c)-e \tilde{v}(h) \\
\text { s.t. } c=e g(h), 0 \leq e \leq 1,0 \leq h \leq 1 .
\end{gathered}
$$

A simple result emerges: in a dynamic model such as this one with nonconvexities, the fraction of life spent in employment becomes a key dimension of labor supply. In fact, in this particular model where individuals do not have any age-varying characteristics, the notion of individual labor supply at a given point in time has no meaning. The only object that has meaning is the lifetime labor supply of the individual, and how this lifetime labor supply is decomposed along the intensive and extensive margins.

As just noted, the model studied here is only able to pin down an individual's lifetime labor supply, not his or her timing of work. ${ }^{6}$ Including life cycle effects such as age-varying productivity or age-varying disutility of work, and considering an overlapping generations model serves to eliminate this indeterminacy. ${ }^{7}$ In this case individuals will choose to work when productivity is highest (or disutility lowest). One is then able to produce well-defined working lives as seen in the data. Specifically, agents enter the labor force at some date and work continuously until retirement, after which time they exit the labor force forever. We abstract from life cycle effects in the remainder of this paper in order to simplify the analysis and focus attention on the key dimension of

\footnotetext{
${ }^{6}$ Mincer (1962) demonstrated a similar result with regard to the labor supply of married women.

${ }^{7}$ Rogerson and Wallenius (2007) construct a model of this nature.
} 
fraction of lifetime devoted to work in the most transparent setting. However, we note that most of our key results continue to hold after the inclusion of life cycle effects.

\section{Policy Analysis}

In this section we use our model to analyze several different policies of interest. The first is a simple tax and transfer scheme that is neutral across ages, in the spirit of Prescott (2004). We then consider policies that directly distort the two key margins of lifetime labor supply, either by legislating the workweek or retirement age, or by specifying social security payments in a manner to target a particular retirement age.

\subsection{Labor Supply Elasticity and Taxes}

In this subsection we analyze the implications of a tax and transfer policy on the length of the standard workweek and the fraction of the lifetime spent in employment. Hence, we now extend the baseline model to include a tax and transfer program. Specifically, we assume that the government taxes all labor income at the constant rate of $\tau$ and uses the tax revenues to fund a lump-sum transfer. The lifetime utility maximization problem can be written as

$$
\begin{aligned}
\max _{c, e, h} & \log (c)-e \tilde{v}(h) \\
\text { s.t. } & c=(1-\tau) \operatorname{eg}(h)+T,
\end{aligned}
$$

where $\tau$ is the tax on labor income and $T$ is the transfer. Government budget balance implies that the transfer must satisfy

$$
T=\tau e g(h) .
$$


The empirically interesting solution to this problem is the interior solution. Hence, this is the case we consider. The first-order conditions for this problem with respect to $e$ and $h$, respectively, are

$$
\begin{aligned}
& \frac{(1-\tau) g(h)}{(1-\tau) e g(h)+T}-\tilde{v}(h)=0 \\
& \frac{(1-\tau) e g^{\prime}(h)}{(1-\tau) e g(h)+T}-e \tilde{v}^{\prime}(h)=0 .
\end{aligned}
$$

From the first-order conditions we can derive expressions that determine the optimal length of the workweek and the fraction of time spent in employment, respectively. We get

$$
\begin{aligned}
& \frac{\tilde{v}^{\prime}(h)}{\tilde{v}(h)}=\frac{g^{\prime}(h)}{g(h)} \\
& e=\frac{1-\tau}{\tilde{v}(h)} .
\end{aligned}
$$

Note that in writing Eqs. (13) and (14), we have utilized the fact that $T=\tau e g(h)$.

Let $h^{*}$ and $e^{*}$ denote equilibrium values in the benchmark model when there is no tax and transfer policy. Let $h_{\tau}$ and $e_{\tau}$ denote the equilibrium values that solve Eqs. (13) and (14).

Proposition 1: $e_{\tau}<e^{*}, h_{\tau}=h^{*}$ and $e_{\tau} h_{\tau}<e^{*} h^{*}$.

Proof of Proposition 1: Eq. (13) determines the optimal length of the workweek. Since $\tau$ does not enter into this equation, the introduction of a tax on labor income, or alternatively an increase in the tax rate, causes no change in the optimal length of the 
workweek. Given $h$, Eq. (14) determines the optimal fraction of time spent in employment. Since there is no change in $h$, an increase in $\tau$ causes a decrease in $e$. Since $e_{\tau}<e^{*}$ and $h_{\tau}=h^{*}$, it follows that $e_{\tau} h_{\tau}<e^{*} h^{*} . / /$

At one level this proposition states the somewhat unsurprising result that a taxtransfer program of the form considered here leads to fewer hours worked in equilibrium. But what is of key importance in this proposition is the result that all of the adjustment in hours takes place along the extensive margin, i.e., the fraction of life devoted to work. This result is of special importance because of its quantitative implications. Specifically, when the extensive margin is the sole margin of adjustment, labor supply is in fact highly elastic. Using Eq. (14) to calculate the elasticity of aggregate hours (eh) with respect to $1-\tau$, we get an elasticity equal to one. Put somewhat differently, the elasticity of hours worked for workers with positive hours, which is equal to zero in this example, provides no information about the magnitude of the aggregate response. A striking implication of this model is that the aggregate labor supply elasticity is a function of technology parameters. Specifically, changing the nonconvexity in the mapping from hours of work to labor services changes the aggregate elasticity. While it is common to think of the aggregate labor supply elasticity as being captured by a preference parameter, as long as the nonconvexity in $g$ is sufficiently large to be operative, the value of the preference parameter governing the disutility from hours of work has no impact on the aggregate elasticity.

\subsection{Constraints on Length of Workweek}

We now analyze the consequences of a binding constraint on workweek length. For the purposes of this analysis we consider the formulation of the individual decision 
problem for both the hours supplied to market formulation as well as the labor services formulation.

From our earlier derivations, recall that once we know the length of the workweek, we also know the optimal fraction of time spent in employment. Specifically, from the hours worked formulation we have the condition

$$
e=\frac{1}{\tilde{v}(h)}
$$

and from the labor service formulation the condition

$$
e=\frac{1}{v(l)}
$$

Let $h^{*}$ and $e^{*}$ denote equilibrium values when there is no hours constraint. Let $\bar{h}$ represent an exogenously imposed hours constraint, and let $e(\bar{h})$ denote equilibrium values in the case of a binding hours constraint. The empirically interesting case is for an interior solution. Hence, we consider the case where $0<e(\bar{h})<1$.

Proposition 2: If $\bar{h}>h^{*}$, then $e(\bar{h})<e^{*}, e(\bar{h}) \bar{h}<e^{*} h^{*}$, and $e(\bar{h}) l(\bar{h})<e^{*} l^{*}$. If $\bar{h}<h^{*}$, then $e(\bar{h})>e^{*}, e(\bar{h}) \bar{h}>e^{*} h^{*}$, and as we make $\bar{h}$ smaller and smaller, first $e(\bar{h}) l(\bar{h})>e^{*} l^{*}$ and then $e(\bar{h}) l(\bar{h})<e^{*} l^{*}$.

Proof of Proposition 2: Given that $\tilde{v}(h)$ is an increasing function, the inverse relationship between $h$ and $e$ is apparent from Eq. (15). Also from Eq. (15), we can easily derive an expression for aggregate hours:

$$
e h=\frac{h}{\tilde{v}(h)} .
$$


To determine the effect of a restriction on $h$ on aggregate hours, the comparative static of interest is

$$
\frac{\partial(e h)}{\partial h}=\frac{\tilde{v}(h)-h \tilde{v}^{\prime}(h)}{\tilde{v}(h)^{2}} .
$$

From the first-order conditions for the problem with no hours restriction we know that $\tilde{v}\left(h^{*}\right)-h^{*} \tilde{v}^{\prime}\left(h^{*}\right)=0$. Recall that $\tilde{v}(h)$ is a convex function, which implies that $\frac{\partial(e h)}{\partial h}<0$. Hence, if $\bar{h}<h^{*}$, then $e(\bar{h}) \bar{h}>e^{*} h^{*}$. As a result, individuals end up with less consumption and less leisure. Conversely, if $\bar{h}>h^{*}$, then $e(\bar{h}) \bar{h}<e^{*} h^{*}$. From Eq. (16) we can easily derive an expression for aggregate efficiency units. In particular, we get

$$
e l=\frac{l}{v(l)} \text {. }
$$

To determine the effect of a restriction on $h$ (which is in essence a restriction on $l$ ) on aggregate efficiency units, the comparative static of interest is

$$
\frac{\partial(e l)}{\partial l}=\frac{v(l)-l v^{\prime}(l)}{v(l)^{2}} .
$$

From the first-order conditions for the case with no restrictions on $l$, we know that $v\left(l^{*}\right)-l^{*} v^{\prime}\left(l^{*}\right)=0$. It is easy to verify that $v(l)-l v^{\prime}(l)$ is monotone decreasing if $v(l)$ is convex. Conversely, $v(l)-l v^{\prime}(l)$ is monotone increasing if $v(l)$ is concave. Recall that $v(l)$ is first concave and then convex. The unconstrained optimum occurs in the convex portion of $v(l)$. Hence, if we restrict the length of the workweek above the optimum, then $e(\bar{h}) l(\bar{h})<e^{*} l^{*}$. If we restrict the length of the workweek somewhat below the optimum (still in convex region), $e(\bar{h}) l(\bar{h})>e^{*} l^{*}$. However, as we restrict the length of the workweek further below the optimum (concave region of $v(l)$ ), $e(\bar{h}) l(\bar{h})<e^{*} l^{*}$. /I 
In the data, it is common to report the hourly wage or the average compensation per hour. Recall that we have normalized the wage per unit of labor services to one. However, the wage per hour of work is different from this measure and is equal to $w^{h}=\frac{\text { income }}{\text { hours of work }}=\frac{g(h)}{h}$. The effect of a restriction on hours of work on the hourly wage is analyzed in the following proposition.

Proposition 3: If $\bar{h}<h^{*}$, then $w^{h}(\bar{h})<w^{h}\left(h^{*}\right)$.

Proof of Proposition 3: The comparative static of interest is

$$
\frac{d w^{h}(h)}{d h}=\frac{g^{\prime}(h) h-g(h)}{h^{2}}
$$

From Eq. (21) it follows that the hourly wage rate will go down as a result of a reduction in hours if $g^{\prime}(h)>\frac{g(h)}{h}$. Recall that the equilibrium condition for hours is $\frac{\tilde{v}^{\prime}(h)}{\tilde{v}(h)}=\frac{g^{\prime}(h)}{g(h)}$. Multiplying both sides by $h$ we get

$$
\frac{\tilde{v}^{\prime}(h)}{\widetilde{v}(h)} h=\frac{g^{\prime}(h)}{g(h)} h .
$$

In order to have $g^{\prime}(h)>\frac{g(h)}{h}$, the right-hand side of Eq. (22) must be greater than one.

This in turn implies that we must have $\tilde{v}^{\prime}(h)>\frac{\tilde{v}(h)}{h}$. Since the $\tilde{v}$ function is convex, this holds. Hence, at least in the neighborhood of the optimum, an upper bound on hours of work will result in a reduction in the hourly wage.// 
A clear prediction of our model is that a decrease in the length of the workweek brings about an increase in the fraction of time spent working or aggregate employment. This has often been the motivation behind government policies that restrict hours worked. However, many empirical studies (see, e.g., Erbas and Sayers 2001) actually find the opposite, i.e., that decreases in the workweek lead to lower employment. In interpreting these studies it is important to note that in practice, policies that restrict workweeks are often accompanied by an increase in the wage rate (e.g., income may be held constant in the face of the decrease in hours worked). Moreover, in our model with the nonlinear mapping from hours to labor services, restrictions on workweeks will actually decrease income per hour worked, since the policy effectively implies an inefficient scale of operation for individual workers.

In related work, Osuna and Ríos-Rull (2003) look for the tax rate on overtime that reduces the workweek from 40 hours to 35 hours. They find that a $12 \%$ tax on overtime work reduces hours of work from 40 to 35 (a reduction of 12.5\%). As a result, employment increases by $7 \%$. While employment does increase, aggregate labor supply (hours times employment) decreases. Their hours measure is in effect an efficiency unit measure. They define hours allocated to work as the total time allotment less leisure and commuting time. Our findings are not contrary to that of Osuna and Ríos-Rull (2003). If a reduction of hours worked from 40 to 35 hours is a sizable enough reduction to move the equilibrium onto the concave portion of $v(l)$, this is exactly what our model focusing on the determination of efficiency units suggests would happen. 


\subsection{Constraints on Working Life}

In our previous analysis of tax and transfer programs, there were no requirements that had to be fulfilled in order for individuals to receive the transfer. We can also design a policy in which individuals must work a certain fraction of their lifetime in order to receive the transfer-namely, $e \geq \bar{e}$. Naturally, we are only interested in the case with a binding constraint on the fraction of time spent in employment. This problem has two possible solutions. Individuals can choose to work the mandated fraction of their time $\bar{e}$, choose the optimal length of the workweek $h(\bar{e})$ given $\bar{e}$, and receive the transfer $T$. Alternatively, they can choose to forgo the transfer and choose $h$ and $e$ without constraints. If individuals choose to receive the transfer payment, they solve the following problem:

$$
\begin{aligned}
\max _{c, e, h} & \log (c)-e \tilde{v}(h) \\
\text { s.t. } & c=(1-\tau) \operatorname{eg}(h)+T . \\
& e \geq \bar{e}
\end{aligned}
$$

Given the constraint on $e$, there is no first-order condition with respect to $e$. Again, the empirically interesting case is for the interior solution with respect to $h$. The first-order condition with respect to $h$ is

$$
\frac{(1-\tau) e g^{\prime}(h)}{(1-\tau) \operatorname{eg}(h)+T}-e \tilde{v}^{\prime}(h)=0 .
$$

Recall that $h(\bar{e})$ denotes the equilibrium value in the case of a binding constraint on $e$.

Proposition 4: If $e=\bar{e}>e_{\tau}$, then $h(\bar{e})<h_{\tau}$. If $e=\bar{e}<e_{\tau}$, then $h(\bar{e})>h_{\tau}$.

Proof of Proposition 4: We can rewrite Eq. (23) as 


$$
\frac{g^{\prime}(h)}{\tilde{v}^{\prime}(h)} \frac{1}{g(h)}=\frac{e}{(1-\tau)} \text {. }
$$

If $e$ increases, the right-hand side of Eq. (24) must also increase. Since $\frac{g^{\prime}(h)}{\tilde{v}^{\prime}(h)}$ and $\frac{1}{g(h)}$ are both decreasing in $h$, when $e$ increases $h$ must decrease. Thus, we have shown that if $e=\bar{e}>e_{\tau}$, then $h(\bar{e})<h_{\tau}$. The second part of the proposition follows by simply reversing the argument. //

Loosely speaking, we can conclude that if the government, for example, raises the retirement age through incentives in social security benefits, people will respond by shortening the length of the workweek.

If individuals choose to forgo the transfer, they solve the following problem:

$$
\begin{aligned}
\max _{c, e, h} & \log (c)-e \tilde{v}(h) \\
\text { s.t. } & c=(1-\tau) \operatorname{eg}(h) .
\end{aligned}
$$

Again, we consider the interior solution to this problem, as it is the empirically interesting one. The first-order conditions for this problem are the same conditions as for the benchmark model with no tax and transfer policy. Hence, $e=e^{*}$ and $h=h^{*}$. When deciding whether or not to forgo the transfer payment, individuals solve

$$
\max \left\{\log ((1-\tau) w \bar{e} g(\bar{h})+T)-\tilde{v}(\bar{h}), \log \left((1-\tau) w e^{*} g\left(h^{*}\right)\right)-\tilde{v}\left(h^{*}\right)\right\},
$$

where to simplify notation we have defined $\bar{h}=h(\bar{e})$.

Similar to the analysis just carried out, if a government imposes a mandatory retirement policy that is binding, then it is easy to show that the result is an increase in hours of work. 


\section{Two-Occupation Example}

In this section we explicitly consider a model with two occupations. There are several motivations for this. First, it allows us to illustrate how our model can account for the differences in workweeks across occupations documented earlier in the paper. Second, in the context of this model we show how empirical exercises that use crossoccupation data to infer labor supply elasticities do not isolate the appropriate elasticity for predicting the effects of the tax and transfer policies considered earlier.

To this end, we extend the baseline model to two occupations. Technological features are a very important distinguishing feature of occupations. Here we abstract from other possible cross-occupation differences. Consequently, here an occupation is defined as a particular $g(h)$ function. We assume that each occupation produces a particular intermediate input. In particular, individuals working with the $g_{1}\left(h_{1}\right)$ technology produce the intermediate input $z_{1}$ and individuals working with the $g_{2}\left(h_{2}\right)$ technology produce the intermediate input $z_{2}$. The intermediate goods technologies are assumed to be linear in labor services, and for convenience we normalize marginal products to unity. We assume that there is one final consumption good, which is produced by aggregating the intermediate goods. In particular,

$$
c=f\left(z_{1}, z_{2}\right)
$$

where $f($.$) exhibits constant returns to scale.$

We assume that the $\tilde{v}($.$) function is the same for individuals in both$ occupations, i.e., that preferences are the same for all individuals.

Initially, we assume that a measure $\mu$ of individuals are endowed with skills that enable them to work in occupation 1 and a measure $1-\mu$ of individuals are endowed 
with skills that enable them to work occupation 2. Later in this section we will endogenize $\mu$.

We normalize the price of the final good to one and let $w_{i}^{l}$ denote the wage rate of a unit of labor services in occupation $i$. The decision problem facing an individual working in occupation $i=1,2$ is

$$
\begin{aligned}
\max _{c_{i}, e_{i}, h_{i}} \log \left(c_{i}\right) & -e_{i} \tilde{v}\left(h_{i}\right) \\
\text { s.t. } \quad c_{i} & =w_{i}^{l} e_{i} g_{i}\left(h_{i}\right) .
\end{aligned}
$$

Again, the empirically interesting case is for an interior solution. After some algebra, the equilibrium conditions for this problem can be written as

$$
\begin{aligned}
& \frac{\tilde{v}^{\prime}\left(h_{i}\right)}{\tilde{v}\left(h_{i}\right)}=\frac{g_{i}^{\prime}\left(h_{i}\right)}{g_{i}\left(h_{i}\right)} \\
& e_{i}=\frac{1}{\tilde{v}\left(h_{i}\right)},
\end{aligned}
$$

where $i=1$,2. Note that the equilibrium conditions that determine $h_{i}$ and $e_{i}$, Eqs. (26) and (27), respectively, are the same as in the case with only one occupation.

To facilitate a comparison of outcomes for individuals in the two occupations, we assume the functional forms $\tilde{v}\left(h_{i}\right)=\frac{h_{i}^{1+\gamma}}{1+\gamma}$ and $g_{i}\left(h_{i}\right)=\left(h_{i}-\phi_{i}\right)^{\eta_{i}}$, where $\eta<1$. Intuitively, we would expect a larger fixed set-up cost to result in a longer optimal workweek length. As demonstrated in the following proposition, this is confirmed by the analytics.

Proposition 5: $h_{i}$ is increasing in $\phi$ and $\eta, e_{i}$ is decreasing in $\phi$ and $\eta$, and $e_{i} h_{i}$ is decreasing in $\phi$ and $\eta$. 
Proof of Proposition 5: Given the functional forms proposed above, we can solve Eq. (26) for $h_{i}$. We get

$$
h_{i}=\frac{1+\gamma}{1+\gamma-\eta_{i}} \phi_{i} .
$$

From this expression it is apparent that $h_{i}$ is increasing in both $\phi$ and $\eta$. We have already previously noted that $e_{i}$ is decreasing in $h_{i}$. It follows that $e_{i}$ is decreasing in both $\phi$ and $\eta$. We know from before that $e_{i} h_{i}$ is decreasing in $h_{i}$. Combining this observation with the fact that $h_{i}$ is increasing in both $\phi$ and $\eta$, we find that $e_{i} h_{i}$ is decreasing in both $\phi$ and $\eta . / /$

As shown in Section 1, workweek lengths differ considerably across occupations. Our framework is able to reconcile this feature of the data.

All our findings regarding the impact of the $g($.) function on the optimal length of the workweek, as well as aggregate hours worked, hold for a general constant returns to scale aggregator $c=f\left(z_{1}, z_{2}\right)$. In the following analysis we will assume a specific functional form for this aggregator. Specifically, $c=z_{1}^{\theta} z_{2}^{1-\theta}$.

Having compared the labor supply outcomes for different occupations, it is of interest to compare the wage rates in the two occupations. As noted previously, the wage rate, $w^{l}$, is the wage rate per unit of labor services. The wage rates reported in the data are per unit of time. Hence, we are interested in comparing the following measures across occupations:

$$
w^{h}=\frac{\text { income }}{\text { hours }}=\frac{w^{l} \cdot \text { units of labor services }}{\text { hours }} .
$$


Both types of individuals are paid the value of their marginal products per unit of labor services produced. This implies that $w_{1}^{l}=p_{1}$ and $w_{2}^{l}=p_{2}$, where $p_{1}$ is the price of $z_{1}$ and $p_{2}$ is the price of $z_{2}$. Hence, the wage rates we are interested in comparing are $w_{1}^{h}=\frac{p_{1} g_{1}\left(h_{1}\right)}{h_{1}}$ and $w_{2}^{h}=\frac{p_{2} g_{2}\left(h_{2}\right)}{h_{2}}$. We can derive an expression for the relative price from the first-order conditions for the final good firm problem. We get

$$
\frac{p_{1}}{p_{2}}=\frac{\theta}{1-\theta} \frac{z_{2}}{z_{1}} .
$$

We know that in equilibrium $z_{1}=\mu e_{1} g_{1}\left(h_{1}\right)$ and $z_{2}=(1-\mu) e_{2} g_{2}\left(h_{2}\right)$. Substituting these into Eq. (30), we get the following wage relation:

$$
\frac{w_{1}^{h}}{w_{2}^{h}}=\frac{\theta}{1-\theta} \frac{1-\mu}{\mu} \frac{e_{2} h_{2}}{e_{1} h_{1}} .
$$

In the preceding analysis we have treated $\mu$ as exogenous. One way to endogenize $\mu$ is to assume that individuals make an occupational choice decision at time zero, and that occupation is fixed thereafter. This would imply that individuals are allocated across the two occupations such that in equilibrium the individuals in the two occupations receive the same utility. This requires that

$$
\log \left(c_{1}\right)-e_{1} \tilde{v}\left(h_{1}\right)=\log \left(c_{2}\right)-e_{2} \tilde{v}\left(h_{2}\right) .
$$

Substituting in for $c_{i}, i=1,2$, from the budget constraint, Eq. (32) reduces to

$$
\log \left(\frac{\theta}{1-\theta} \frac{1-\mu}{\mu}\right)=e_{1} \tilde{v}\left(h_{1}\right)-e_{2} \tilde{v}\left(h_{2}\right)
$$

Equation (27) further implies that $\log \left(\frac{\theta}{1-\theta} \frac{1-\mu}{\mu}\right)=0$, which in turn implies that $\theta=\mu$. In other words, input shares determine the distribution of individuals across occupations. 
As a result, individuals in the two occupations enjoy the same consumption. Naturally this implies that incomes across the two types of individuals are equalized. Note, however, that wages are not equal across occupations. Given that $\theta=\mu$, we have

$$
\frac{w_{1}^{h}}{w_{2}^{h}}=\frac{e_{2} h_{2}}{e_{1} h_{1}} .
$$

Recall that if $h_{1}>h_{2}$, then $e_{1} h_{1}<e_{2} h_{2}$. Hence, we can conclude that the occupation with the longer workweek will have a higher wage.

We now consider the implications of this analysis for uncovering labor supply elasticities using cross-section data. Given Eq. (27) and our assumed functional forms, we can write the relative wage as

$$
\frac{w_{1}^{h}}{w_{2}^{h}}=\left(\frac{h_{1}}{h_{2}}\right)^{\gamma} \text {. }
$$

Taking logs we can rewrite this expression in the more standard way:

$$
\log \left(\frac{h_{1}}{h_{2}}\right)=\frac{1}{\gamma} \log \left(\frac{w_{1}^{h}}{w_{2}^{h}}\right) .
$$

This expression implies that if one looks at the covariation of wages and hours, one will get an estimate of the curvature parameter $\gamma$. Empirical studies based on cross-section data typically find this parameter to be quite small, i.e., that $\gamma$ is relatively large (see, e.g., the survey article of Pencavel, 1986). However, what is interesting to note is that if we analyze a tax and transfer scheme in this two-occupation economy similar to the one considered earlier in the single occupation model, one obtains exactly the same results as before. Specifically, workweeks remain constant and all adjustment occurs along the extensive margin. This implies that the value of $\gamma$ is actually irrelevant in determining the magnitude of the decrease in aggregate hours of work. 
In a model with identical individuals in which occupations are equally costly to enter, our analysis delivers sharp predictions about workweeks and working lives. One can note that if $\mu$ is taken as exogenous, perhaps because some individuals do not have the skill necessary to enter, these results may be affected. Additionally, in this case one cannot infer the preference parameter $\gamma$ from cross-section data without knowing something about how these costs differ across occupations. To see this, we now extend the preceding analysis to the case where there is a fixed utility cost associated with entering each occupation. We allow the utility cost to differ across occupations. One interpretation for this cost is the time individuals give up or the consumption they forgo in order to train for an occupation. The utility cost $m_{i}$ enters the decision problem of consumer $i$ in the following way:

$$
\begin{array}{r}
\max _{c_{i}, e_{i}, h_{i}} \log \left(c_{i}\right)-e_{i} \tilde{v}\left(h_{i}\right)-m_{i} \\
\text { s.t. } \quad c_{i}=w_{i}^{l} e_{i} g_{i}\left(h_{i}\right) .
\end{array}
$$

After some algebra, we can write the equivalent of Eq. (32) as

$$
\log \left(\frac{\theta}{1-\theta} \frac{1-\mu}{\mu}\right)=m_{1}-m_{2} .
$$

Given this expression, we can show that $\mu>\theta$ if $m_{1}<m_{2}$. If this is the case, $c_{2}>c_{1}$. That is, individual 2 receives more of the consumption good in order to compensate for the higher fixed utility cost. The expression for the relative wage becomes

$$
\frac{w_{1}^{h}}{w_{2}^{h}}=\exp \left(m_{1}-m_{2}\right) \frac{e_{2} h_{2}}{e_{1} h_{1}} .
$$

Again, using Eq. (27) and our specified functional forms, we can rewrite this as

$$
\frac{w_{1}^{h}}{w_{2}^{h}}=\exp \left(m_{1}-m_{2}\right)\left(\frac{h_{1}}{h_{2}}\right)^{\gamma} .
$$


This analysis cautions against using the covariation of wage and hours to infer the magnitude of $\gamma$ without knowing the magnitudes of the fixed utility costs.

\section{Conclusion}

Lifetime labor supply of an individual is usefully characterized by two key numbers: the fraction of life spent in employment, and the hours of work while employed. We build a model that delivers this characterization as an equilibrium outcome. A nonconvexity in the mapping from hours of work to labor services provided is key to this prediction. The model represents a significant simplification over previous formulations. We consider the qualitative effects of several policy changes to illustrate both the tractability of the formulation and its ability to capture important economic forces. Life cycle effects can easily be added to the framework presented here (see Rogerson and Wallenius, 2007), further illustrating the usefulness of this abstraction. 


\section{References}

Cho, Jang-Ok and Thomas F. Cooley. 1994. "Employment and Hours over the Business Cycle.” Journal of Economic Dynamics and Control 18 (March): 411-432.

Erbas, S. Nuri and Chera L. Sayers. 2001. "Can a Shorter Workweek Induce Higher Employment? Mandatory Reductions in the Workweek and Employment Subsidies.” International Tax and Public Finance 8 (August): 485-509.

Hornstein, Andreas and Edward C. Prescott. 1993. “The Firm and the Plant in General Equilibrium Theory.” In General Equilibrium, Growth, and Trade II: The Legacy of Lionel McKenzie, edited by Robert Becker, Michele Boldrin, Ronald Jones, and William Thomson. San Diego: Academic Press.

Mincer, Jacob. 1962. “Labor Force Participation of Married Women.” In Aspects of Labor Economics, edited by H. Gregg Lewis. Princeton, NJ: Princeton University Press.

Osuna, Victoria and José-Víctor Ríos-Rull. 2003. “Implementing the 35 Hour Workweek by Means of Overtime Taxation.” Review of Economic Dynamics 6 (January): 179-206.

Pencavel, John H. 1986. “Labor Supply of Men: A Survey.” In Handbook of Labor Economics, vol. 1, edited by Orley Ashenfelter and Richard Layard. Amsterdam: North-Holland, pp. 3-102.

Prescott, Edward C. 2004. "Why Do Americans Work So Much More than Europeans?” Federal Reserve Bank Minneapolis Quarterly Review 28 (July): 2-13. 
Prescott, Edward C. and Robert M. Townsend. 1984a. "General Competitive Analysis in an Economy with Private Information.” International Economic Review 25 (February): 1-20.

Prescott, Edward C. and Robert M. Townsend. 1984b. "Pareto Optima and Competitive Equilibria with Adverse Selection and Moral Hazard.” Econometrica 52 (January): 21-45.

Rogerson, Richard. 1988. "Indivisible Labor, Lotteries and Equilibrium.” Journal of Monetary Economics 21 (January): 3-16.

Rogerson, Richard and Johanna Wallenius. 2007. "Micro and Macro Elasticities in a Life Cycle Model with Taxes.” Working Paper 13017. National Bureau of Economic Research.

Rosen, Sherwin. 1978. “The Supply of Work Schedules and Employment.” In Work Time and Employment. Washington, DC: National Commission for Manpower Policy.

Shell, Karl and Randall Wright. 1993. "Indivisibilities, Lotteries, and Sunspot Equilibria.” Economic Theory 3 (January): 1-17. 